\section{IMMUNOLOGIC TUMOR CELL INTRINSIC EFFECTS OF STANDARD OF CARE THERAPIES FOR OVARIAN CANCER}

${ }^{1}$ Nicole James*, ${ }^{2}$ Melih Ozsoy, ${ }^{2}$ Payton De La Cruz, ${ }^{1}$ Morgan Woodman, 'Jennifer Ribeiro. ${ }^{1}$ Women and Infants Hospital, Providence, RI, United States; ${ }^{2}$ Brown University, Providence, RI, United States

Background Outcomes for high grade serous ovarian cancer (HGSOC) patients have remained dismal due to the inevitable development of chemotherapy resistance with recurrent disease. ${ }^{1}$ In order to better tailor treatment approaches and uncover opportunities for novel treatments, we need to better understand factors contributing to chemotherapy resistance. Recent studies have shown that immune-related gene expression profiles may serve as prognostic indicators of response to chemotherapy and clinical outcomes in solid tumors, including ovarian cancer. ${ }^{2-7}$ Moreover, immunologic factors have been shown to mediate chemotherapy resistance ${ }^{8}$ Reports in the literature show that common ovarian cancer therapeutics, including chemotherapy, PARP inhibitors, and bevacizumab, modulate tumor cell expressed PD-L1 levels through immunologic signaling pathways. ${ }^{9-12}$ However, very little research has addressed the effect of these treatments on other immune ligands or the differences in immunologic responses between platinum-sensitive and platinum-resistant HGSOC cell lines.

Methods The HGSOC cell lines OVCAR4 (naturally platinumresistant), PEO1 and PEO4 (matched platinum-sensitive and resistant lines from the same patient), were treated with common ovarian cancer therapeutics (carboplatin/paclitaxel, olaparib, and bevacizumab), in the presence or absence of peripheral blood mononuclear cells. Western blot was employed to identify levels of immune ligands of interest and a proteome profiler was used to detect broad immunologic changes in response to standard of care therapeutics.

Results Olaparib and bevacizumab treatment strikingly upregulated levels of tumor cell expressed immune ligands ICOSL and PVRL2. Platinum status or presence of an immune component had no bearing on the effect. Moreover, blockade of PVRL2 using siRNA or monoclonal antibodies suppressed STAT3 signaling. When examining the effect of these therapeutics on cytokine levels in HGSOC cell lines treated in immune cell co-culture, OVCAR4 cells displayed marked changes in cytokine levels, particularly CXCL10, CXCL12, SERPINE1, IL1A, and IL1RA. While PEO1 and PEO4 cells displayed more subtle cytokine changes compared to OVCAR4 cells, differences in basal levels and treatment responses were observed between the platinum-sensitive and -resistant lines, most strikingly higher basal levels of SERPINE1 and CCL5/ RANTES in PEO4 cells, and a robust increase in IL8 levels in response to chemotherapy in only PEO1 cells and not PEO4.

Conclusions In conclusion, common ovarian cancer chemotherapeutics and targeted agents induce tumor cell intrinsic immunologic effects that could potentially be exploited as combinatorial therapeutic targets. Differences in immunologic responses may help define platinum-sensitive and -resistant disease. These results will require further exploration in immunecompetent mouse models and human HGSOC tissue.

\section{REFERENCES}

1. Cortez AJ, Tudrej P, Kujawa KA, Lisowska KM. Advances in ovarian cancer therapy. Cancer Chemother Pharmacol 2018;81(1):17-38.

2. James NE, Miller K, LaFranzo N, Lips E, Woodman M, Ou J, Ribeiro JR. Immune modeling analysis reveals immunologic signatures associated with improved outcomes in high grade serous ovarian cancer. Front Oncol 2021;11:622182.

3. Liu R, Hu R, Zeng $Y$, Zhang $W$, Zhou H-H. Tumour immune cell infiltration and survival after platinum-based chemotherapy in high-grade serous ovarian cancer subtypes: a gene expression-based computational study. EBioMedicine 2020:51:102602

4. Liu J, Meng H, Nie S, Sun Y, Jiang P, Li S, et al. Identification of a prognostic signature of epithelial ovarian cancer based on tumor immune microenvironment exploration. Genomics. 2020.

5. Ding J, Zhang Q, Chen S, Huang H, He L. Construction of a new tumor immunity-related signature to assess and classify the prognostic risk of ovarian cancer. Aging (Albany, NY). 2020;12.

6. Wu Y, Xia L, Zhao P, Deng Y, Guo Q, Zhu J, et al. Immune profiling reveals prognostic genes in high-grade serous ovarian cancer. Aging (Albany, NY). 2020;12(12):11398-11415.

7. Montfort A, Owen S, Piskorz AM, Supernat A, Moore L, Al-Khalidi S, et al. Combining measures of immune infiltration shows additive effect on survival prediction in high-grade serous ovarian carcinoma. Br J Cancer 2020;122(12):1803-1810.

8. Liu W, Wang Y, Xie Y, Dai T, Fan M, Lu C, Zou Y. Cisplatin remodels the tumor immune microenvironment via the transcription factor $E B$ in ovarian cancer. Cell Death Discov. 2021;7(1):136.

9. Peng J, Hamanishi J, Matsumura N, Abiko K, Murat K, Baba T, Yamaguchi K, Horikawa N, Hosoe Y, Murphy SK, Konishi I, Mandai M. Chemotherapy induces programmed cell death-Ligand 1 overexpression via the nuclear factor- $\mathrm{KB}$ to foster an immunosuppressive tumor microenvironment in Ovarian cancer. Cancer Res 2015;75(23):5034-45.

10. Jiao S, Xia W, Yamaguchi H, Wei Y, Chen M-K, Hsu J-M, et al. PARP inhibito upregulates PD-L1 expression and enhances cancer-associated immunosuppression. Clin Cancer Res 2017;23(14):3711-3720.

11. Xue C, Xu Y, Ye W, Xie Q, Gao H, Xu B, et al. Expression of PD-L1 in ovarian cancer and its synergistic antitumor effect with PARP inhibitor. Gynecol Oncol 2020;157(1):222-233.

12. Zhang L, Chen Y, Li F, Bao L, Liu W. Atezolizumab and bevacizumab attenuate cisplatin resistant Ovarian cancer cells progression synergistically via suppressing epithelial-Mesenchymal transition. Front Immunol 2019;10:867.

http://dx.doi.org/10.1136/jitc-2021-SITC2021.729 